\title{
El despliegue conjunto de la dualización productiva normal: desde su inicio hasta el uso primero del producto. Bases antropológicas próximas de la actividad económica (IV)
}

\author{
The Conjoint Unfolding of The Normal Productive \\ Dualization: From Its Beginning to The First Use of \\ The Product. The Proximate Anthropological Bases \\ of Economic Activity (IV)
}

RECIBIDO: 24 DE DICIEMBRE DE 2021/ ACEPTADO: 31 DE ENERO DE 2022

\section{IGNACIO FALGUERAS SALINAS \\ Catedrático Emérito de Filosofía \\ Universidad de Málaga \\ jifalgueras@uma.es \\ ORCID 0000-0002-5664-0141}

\author{
IGNACIO FALGUERAS SORAUREN \\ Profesor Contratado Doctor, Departamento de \\ Teoría e Historia Económica \\ Universidad de Málaga \\ ifs@uma.es \\ ORCID 0000-0003-0367-1523
}

Resumen. Estudiamos aquí el primer tramo del proceso productivo, común a toda producción humana, y que permite comprenderla mejor. Dicho proceso se inicia al ejecutar las facultades locomotrices el proyecto elegido; reviste la forma del método «ensayo-error-nuevo ensayo...», y alcanzará su primer objetivo al conseguir un producto suficientemente satisfactorio para la idea a plasmar. Sin embargo, este primer objetivo no cumple por completo el propósito inicial del proceso, que sólo se alcanza cuando el producto es usado para lo pretendido. Justificada la noción de uso, la consideración del primero de ellos sirve para establecer la noción básica de utilidad.

Palabras clave: Producción, Producto, Utilidad, Antropología de Leonardo Polo.
Abstract. We analyze the first stretch of the productive process, which is common to all forms of human production and enhances our understanding of them. This process begins when the locomotive faculties implement the chosen project; develops according to the «trial-error-new trial... » method; and will achieve its immediate goal when a product that conforms closely enough to the guiding idea is obtained. However, this first step does not accomplish the initial purpose of the productive process, which will only be attained when the product is used. Once the notion of use is introduced, the elemental notion of utility is derived from the consideration of the first type of use of the product.

Keywords: Production, Product, Utility, Leonardo Polo's Anthropology. 


\section{INTRODUCCIÓN}

Cuando se trata de estudiar la repercusión que la propuesta antropológica de Polo tiene en los ámbitos de la economía y la dirección de empresas caben, al menos, dos opciones, ambas igualmente legítimas, pero que se diferencian por el calado filosófico de los resultados obtenidos. Por un lado, es posible buscar la aplicación directa de su pensamiento a problemas o cuestiones actuales (prácticas o teóricas) en ambos campos, aprovechando para ello todo lo que al respecto dejó escrito -que, especialmente en el ámbito de la dirección de empresas, fue bastante-. Este camino, sin duda necesario, tiene la ventaja de que produce resultados inmediatamente visibles, aunque a costa de no exprimir toda la riqueza de su propuesta filosófica, por lo que el alcance de los hallazgos así logrados deja cuestiones antropológicas de fondo sin resolver. Por otro lado, existe una vía alternativa que enlaza su antropología filosófica con las cuestiones y temas primeros de la economía y de la dirección de empresas, a los que suele dedicársele menos atención, pero que son decisivos a la larga. Esta ruta es más ardua e intrincada que la anterior, y sus primeros resultados no tienen una aplicación práctica inmediata. Sin embargo, ella tiene la gran ventaja de que permite proseguir la propuesta filosófica de Polo más allá de donde él la dejó, a la par que ayuda a iluminar y dar una respuesta más integral y adecuada a las cuestiones antropológicas radicales que la actividad económica sigue suscitando en nuestros días.

Nosotros nos hemos decantado por la segunda vía, de modo que, en nuestra contribución, siguiendo la línea de anteriores investigaciones, continuaremos el estudio del despliegue conjunto de la dualización productiva nor$\mathrm{mal}^{1}$, que es el punto en que nos habíamos detenido. Conviene aclarar que las dualizaciones no son estáticas, sino que se despliegan en procesos crecientes o decrecientes. Y son así porque la dualización es una característica distintiva de la persona humana, que está vinculada existencialmente con el ser del mundo, y se manifiesta en su esencia dualizándose con la esencia del mundo a través de su corporalidad. Según esto, somos duales con el mundo, pero no en igualdad, sino en superioridad, al ser nosotros los que unilateralmente tomamos la iniciativa y nos dualizamos activamente con él. Por ello, toda dualización está integrada por dos polos de diferente altura que intervienen conjuntamente en

1 Según hemos explicado en Falgueras Salinas, I. y Falgueras Sorauren, I. (2016), pp. 44-45, por «normal» se entiende la dualización productiva más equilibrada, pero tal como nosotros la conocemos en nuestra actual situación, es decir, después del pecado de origen. 
unidad de acción, pero cada uno de manera diferente: la iniciativa parte siempre del polo superior, mientras que el inferior interviene poniendo condiciones para ser asociado a dicha iniciativa. Cuando el polo superior asume activamente las condiciones que impone el inferior, lo asocia a su actividad obteniendo un resultado operativamente conjunto. Ahora bien, esa acción conjunta no puede ser entendida como si fuera puramente atemporal (espiritual) ni puramente temporal (corporal), sino que, dada tal complejidad, su estudio se ha de distribuir en partes.

Concretamente, en la dualización esencial productiva -que también hemos llamado «producción» $\mathrm{O}$ 《actividad productiva» ${ }^{2}-$ hemos distinguido entre la fase personal-individual y la personal-social, no porque estén ellas separadas entre sí, sino porque, al integrarse en dualización, se distienden en el tiempo, pero manteniendo su unidad desde fuera de él, y dando lugar a un proceso creciente o decreciente ${ }^{3}$. Naturalmente, por más que ambas fases formen parte de un mismo proceso, en el que están unidas, siempre es posible y conveniente hacer, primero, un estudio de ellas por separado, para intentar, después, entender sin confusiones su funcionamiento conjunto.

Hemos dedicado ya sendos artículos a la consideración por separado de cada uno de los dos polos que integran la dualización referida en su fase personal-individual, que es a la que, de acuerdo con lo anteriormente indicado,

2 Por «producción» entendemos la introducción de una idea en el tiempo, o bien la ordenación de procesos físicos según una idea (cfr. Falgueras Salinas, I. y Falgueras Sorauren, I. (2020), p. 138, nota 14). Se trata de una noción más amplia y abarcante que la usual, pues incluye la noción de trabajo en sus dos facetas -de esfuerzo penoso, y de vocación inspiradora, cfr. nota 68 del presente trabajo-, y, además, no excluye el trabajo considerado, por los economistas, como improductivo -v.gr.: hablar, bailar, etc.-, ni separa lo técnico de lo ético. En suma, por ser -antropológicamente hablando- más básica, esta noción abarca todos los sentidos usuales. Lo cual no implica que en ella hayan de confundirse las diferencias, por ejemplo, entre lo técnico y lo ético, tan sólo significa que, tal como aquí la entendemos, la «producción» es común a todas las dimensiones de la actividad práctica humana, en este sentido tendría cierta afinidad con la concepción de la acción humana en la praxiología (cfr. Von Mises, L., 1996).

3 Cfr. Falgueras Salinas, I. y Falgueras Sorauren, I. (2020), pp. 154-155. El término «fase» es sinónimo de «etapa» dentro de un movimiento o proceso continuo. Y, puesto que lo que consideramos aquí es el funcionamiento conjunto de la atemporalidad del alma y la temporalidad del cuerpo, alguien podría objetar que más que de «fases», de lo que se habla aquí es de «estratos» o «capas» dentro de una dualización en la que tanto lo personal-individual como lo personal-social se entrelazan sin confundirse. Sin embargo, la noción de «estrato» es espacial, no temporal, por lo que no sería posible usarla aquí más que, metafóricamente, para señalar una diferencia en jerarquía. En cambio, como lo personal-individual no es diferente en jerarquía respecto de lo personal-social, pero sí tiene prioridad de iniciativa en el proceso dualizante, es apropiado considerarlo como una etapa o fase del mismo. 
le corresponde la iniciativa ${ }^{4}$. Y en ella el primer polo estudiado -de modo sólo preponderante- fue el inferior y negativo, a saber, la escasez de tiempo, derivada de la índole corporal y «caída» de nuestra esencia ${ }^{5}$. Posteriormente, el segundo polo estudiado -también de modo sólo preponderante- ha sido el polo positivo y superior, a saber, la riqueza, constituida primero por las posesiones inmanentes del espíritu, y, después, por las transitivas del cuerpo ${ }^{6}$.

El paso obligado a continuación era el de estudiar el despliegue conjunto de ambos polos. Lo mismo que -si se nos permite una metáfora platónica- el nacimiento de Eros (amor apasionado) depende del concurso de Poros (riqueza de habilidades) y de Penía (indigencia) ${ }^{7}$, así la habitación del mundo por el hombre, en su situación actual, depende de la actividad atemporal de su alma (riqueza) y de la cooperación temporal de su cuerpo (escasez). Dicho de otro modo, los dos polos han de concurrir en unidad para integrar la actividad productiva conjunta, por la que empieza en el mundo toda acción humana ${ }^{8}$.

Naturalmente, la actividad productiva humana puede alcanzar una complejidad muy alta ${ }^{9}$, pero en todos los casos contiene un núcleo inicial, del cual parte. Dicho núcleo está integrado por el enlace activo de lo atemporal (ideas y voliciones) y de lo temporal (procesos físicos) del obrar humano ${ }^{10}$. Y, aunque está contenido en cualquier intervención humana en el mundo, es más sencillo observarlo y estudiarlo en los inicios de su despliegue ${ }^{11}$. Precisamente por ser el núcleo de cada acción humana en el mundo, es la actividad productiva conjunta en su fase personal-individual la que ha servido de guía y ha calificado,

4 La fase personal-individual es la primera, porque la razón de la dualización está en la persona humana en cuanto que es (i) irrestricta y (ii) comunicativa, y corresponde a la irrestricción, mientras que la personal-social corresponde a la dimensión comunicativa (a través del cuerpo), por lo que viene después de aquélla.

5 Cfr. Falgueras Salinas, I. y Falgueras Sorauren, I. (2020), pp. 156 y ss.

6 Cfr. Falgueras Salinas, I. y Falgueras Sorauren, I. (2022a).

7 Platón (1903), pp. 203-204.

8 Los términos «actividad productiva», «proceso productivo», «dualización productiva» son aquí sinónimos precisamente porque se refieren a lo que es común a toda actividad práctica humana: la introducción de una idea en el tiempo físico, o producción en sentido amplio. Todos ellos tienen como opuesto el pensamiento teórico, no el descanso, la diversión o el tiempo libre, pues -menos teorizar, o dormir- cuanto se haga durante el ocio también será producción en sentido amplio.

9 Piénsese, por ejemplo, en la calidad y cantidad de ideas y de personas que se requieren para enviar al espacio un telescopio como el James Webb.

10 Acerca de la integración de lo atemporal y lo temporal de la acción humana, así como del papel que en ella juega la imaginación. cfr. Falgueras Salinas, I. y Falgueras Sorauren, I. (2022b).

11 Es lo que pasa, v.gr., con la «regla de tres», que, como decía Espinosa, no es evidente en los grandes números, pero puede ser intuida fácilmente en los primeros números -1 es a 2, como 3 es a 6 ; y 2`3/1=6-(Espinosa, B., 1972, II, Prop. 40, Scholium II, p. 122). 
en nuestra investigación, al despliegue entero de la esencia humana, o sea, a la dualización normal como productiva. Debe notarse, sin embargo, que, al ampliar su estudio en este trabajo, adquiere un sentido más restringido y se refiere sólo al núcleo básico de toda la dualización productiva esencial ${ }^{12}$.

En esta investigación, pues, consideraremos tal despliegue conjunto -de los dos polos referidos, riqueza de posesiones y escasez de tiempo- en la fase personal-individual, pero sólo en su comienzo: desde la iniciación de la actividad productiva conjunta hasta el primer uso del producto ${ }^{13}$. Empezaremos por describir el arranque del proceso productivo conjunto (I): desde el inicio hasta la «consecución» del producto (I.1), para describir, después, la doble dimensión (externa e interna) que le corresponde a dicha «consecución», con especial atención al producto externo (I.2). Examinaremos, más tarde, el uso primero del producto externo con el que se completa inicialmente la actividad productiva conjunta (II): concretando, a la vez, las nociones primitivas de uso (II.1) y de utilidad (II.2). Cerraremos el trabajo con un resumen de los resultados obtenidos (III).

\section{DESCRIPCIÓN DEL INICIO DEL PROCESO PRODUCTIVO CONJUNTO}

\section{Desde su iniciación hasta la consecución del producto}

Todo inicio que no es un comienzo puro ha de tener unos antecedentes que preparen la aparición de la relativa novedad que él aporta. Téngase en cuenta que de lo que hablamos es de un proceso, o sea, de una actividad que se desarrolla mediante una sucesión de actos. Sin embargo, aquí empezaremos

12 Pero, incluso en este sentido restringido, la noción de producción que aplicamos aquí no coincide con la que se suele tener en Economía -la transformación de factores productivos en producto (cfr. Rasmussen, S., 2013, p. 1)-, y siempre habida cuenta de que en la disciplina económica la producción se analiza en asociación con las leyes de intercambio (cfr. Wicksell, K. (1938), I, p. 102). Sostenemos que la noción de producción propuesta por nosotros es más básica, porque -sin excluir la económica, más compleja-: (i) alude a una actividad humana anterior y más amplia que la producción para el intercambio, y (ii) es más que un mero factor productivo, como propone Rasmussen, al referirse ella a aquella actividad que integra los factores productivos para que lo sean, por lo que -en esa medida- es prioritaria y más determinante que ellos en la producción.

13 Este fraccionamiento (teórico) no daña al contenido de nuestra investigación, pues toda la actividad práctica humana está sometida a una obligada fragmentación (cfr. Polo, L. (2015b), p. 268; Falgueras Salinas, I. (2020), pp. 49-54). 
por atender sólo al comienzo mismo del proceso productivo, dado que la situación anterior del despliegue ya ha sido considerada por nosotros en otro trabajo precedente ${ }^{14}$.

La iniciación de la dualización productiva normal siempre tiene lugar cuando: (i) las facultades locomotrices empiezan a introducir en el tiempo físico (ii) la idea de un proyecto humano, (iii) por mandato de la razón práctica y (iv) con ayuda de la imaginación, que proporciona detalles sobre los procesos físicos en los que introducirlas, y va sugiriendo el modo idóneo de dicha introducción. Gracias, pues, a la mediación imaginativa se puede iniciar la actividad conjunta de alma y cuerpo, o sea, la dualización productiva, que por eso mismo puede considerarse como la más equilibrada del hombre, y como la normal en su situación actual.

Pero si nos fijamos en el inicio que acabamos de describir, podemos constatar que el proceso empieza en el orden físico cuando intervienen conjuntamente: (i) las potencias espirituales -inteligencia (ideas), voluntad (elección y moción), que integran la razón práctica-; (ii) las potencias corporales -sensibles externas (cinco sentidos) e internas (sentido común, imaginación, memoria y cogitativa)-, así como las facultades locomotrices. Justo al intervenir todo ese conjunto de potencias se pone en marcha la actividad productiva. De este modo tan complicado empieza lo que llamamos el despliegue conjunto de la dualización esencial normal, en lo cual va dicho que toda intervención normal humana en el mundo tiene su inicio en la «producción» estricta, entendida ésta como la referida integración activa de las potencias espirituales y corporales para introducir una idea en el tiempo físico, u ordenar el tiempo físico según una idea.

Pues bien, el proceso que así se pone en marcha ha de superar la diferencia entre los dos tipos de objetivaciones, unas temporales (sensitivas) y otras atemporales (ideas), que se conjugan en ella. Se comprende que sólo se podrá entrelazarlas reajustando sucesiva y recíprocamente tanto la ordenación atemporal del tiempo según las ideas, como la temporalización física de lo atemporal. Tales reajustes hacen patente la importancia funcional de la imaginación en la conjunción operativa de alma y cuerpo ${ }^{15}$. Pero la necesidad de tales reajustes implica que hemos de empezar siempre con lo que se suele llamar el

14 Falgueras Salinas, I. y Falgueras Sorauren, I. (2022b), del que es continuación el presente artículo.

15 Cfr. Falgueras Salinas, I. y Falgueras Sorauren, I. (2022b). 
«método ensayo-error-corrección... ${ }^{16}$ : se intenta hacer algo, y, tras introducir la idea proyectada en los procesos físicos, se corrige repetidamente el modo concreto de introducción hasta conseguir la eficacia deseada en lo producido.

Ahora bien, al tener que conjuntar la actividad de todas las potencias referidas, introducir un proyecto en el mundo físico siempre ha de llevar tiempo. Este «llevar tiempo» se debe, en primer lugar, a lo que hemos llamado en otra ocasión «restricciones temporales», pues -aunque en el proyecto la idea y la voluntad estén activas de una vez, o sea, sin consumir tiempo- ya el imaginar adecuadamente su introducción en los procesos físicos requiere un primer retraso respecto de lo ideal, a saber: el exigido para distribuir la idea según el movimiento de la imaginación, así como según el antes y el después de la memoria y de la cogitativa, de manera que la quietud de la idea se pueda acomodar al transcurrir extramental. A eso se añade el retraso en tiempo físico externo que lleva consigo el empleo de las facultades locomotrices en la ejecución del proyecto, y que suele ser, sobre todo al principio, bastante notable. Y, finalmente, lleva tiempo porque ha de hacerse -como ya hemos visto- por pasos temporalizados, o, lo que es equivalente, procediendo de modo acompasado según el método «ensayo-error-corrección-ensayo...», y pudiendo siempre quedar interrumpido o malogrado en cualquiera de ellos ${ }^{17}$.

Resumiendo, el mencionado gasto de tiempo deriva de la inclusión de procesos físico-temporales ${ }^{18}$ en el proceso productivo humano. Es importante notar la diferencia entre los mencionados procesos temporales y el proceso productivo. En efecto, el proceso productivo es un proceso libre: (i) que tiene un comienzo libre ${ }^{19}$, (ii) que puede interrumpirse y retomarse ${ }^{20}$, (iii) que tiene un término pre-definido ${ }^{21}$. En cambio, los procesos físico-temporales son mo-

16 Cfr. Inciarte, F. (1974), p. 187.

17 Por tales razones, estos ensayos, en cuanto que no llegan a un término satisfactorio, pueden ser considerados como «conatos» de producto.

18 En el mundo extramental todo está en movimiento y, por tanto, en continua variación (cfr. Falgueras Salinas, I. (2021), I, pp. 297-298).

19 Se trata del imperium de la razón práctica, cfr. Falgueras Salinas, I. y Falgueras Sorauren, I. (2022a).

20 Gracias a la mayor o menor velocidad de movimiento de los procesos físicos, el hombre puede servirse de unos para controlar a los otros. Al tener un fin libremente designado por el hombre, la producción no está sometida a la necesidad final, que es probabilística o estadística, y rige en el mundo físico. Por eso, cabe interrumpir el proceso físico-temporal sin que eso afecte de modo necesario al proceso productivo.

21 Está predefinido en el propio proyecto humano, el cual contiene el fin al que apunta. 
vimientos continuos ${ }^{22}$ : (i) que no tienen un comienzo definido ${ }^{23}$, (ii) que cesan si se interrumpen ${ }^{24}$, y (iii) que no tienen un término definido. Al ser tan distintos, la fusión entre ambos procesos sólo puede ser lograda si existe entre ellos una diferencia jerárquica -implícita en la descripción recién hecha-que permita al proceso productivo dominar los procesos temporales, asignándoles fines propios.

Con todo, la acumulación de todos los retrasos señalados alarga en el tiempo, inevitablemente, el intento de llevar a término de manera satisfactoria el proceso productivo. Y como la producción antecede al producto, pero ha de proceder ensayando, se debe distinguir entre los términos de cada ensayo, y la obtención del producto. Sólo se puede considerar (relativamente) acabado el proceso productivo cuando se obtiene un producto que dote de suficiente efectividad a la idea del productor, mientras que los términos de los ensayos previos han de considerarse, más bien, «conatos» de producto. En este sentido, llamamos «consecución» a la terminación satisfactoria para el proyecto del productor de su actividad productiva, la cual se alcanza cuando se obtiene un resultado adecuado a la idea que él intentaba plasmar de modo inmediato en el producto.

22 El pedernal, por ejemplo, lo puede encontrar el hombre como un canto rodado, pero obviamente esa forma de pedernal no es la primera ni será la última que tengan sus componentes: éstos existen desde el comienzo del universo y durarán hasta su final, sólo que en composiciones que varían incesantemente. Antes de su forma de canto rodado debió ser parte de alguna montaña formada por una erupción volcánica y afectada por movimientos tectónicos hasta que la erosión le ha dado la forma en que lo encontramos. Pero esa no es su última forma, pues la propia erosión a lo largo de millones de años la convertirán en arena, y la arena se convertirá en polvo o sedimentos; y podemos pensar que cuando el sistema solar llegue a desaparecer sus componentes se habrán vuelto a convertir en energía, por ejemplo, y seguirán recibiendo otras formas. El pedernal no «es» una substancia quieta y estable, sino un momento de un proceso temporal físico.

23 Hablamos aquí de los movimientos de la esencia del mundo. Y no decimos que no tengan comienzo, sino que su comienzo no es necesario con necesidad de causa eficiente, sino sólo con necesidad de causa final, la cual es compatible con el azar. En ese sentido, decimos que su comienzo no es definido. El único comienzo definido de todo movimiento es el ser del mundo, o principio de contradicción, o movimiento puro, el cual nunca termina o cesa. Decimos, en cambio, que los movimientos esenciales no tienen un término definido, porque, si bien cesan o tienen término, su cese puede ocurrir por cualquiera de las causas o por azar.

24 Esa es una característica distintiva de todo movimiento: sólo existe en tanto que, una vez comenzado, no cesa. 


\section{La «consecución» y su doble resultado (externo e interno)}

La «consecución» del proceso productivo tiene, por su parte, una doble vertiente, a saber: un resultado físico externo (el producto), y un resultado interno a la propia actividad introductiva, o sea, a la producción.

El término primero del proceso productivo ${ }^{25}$ no se reduce al resultado externo en el que cesa transitoriamente, sino que está acompañado de un aumento de la capacidad para introducir lo ideado y para ordenar -según la idea contenida en el proyecto- determinados procesos físicos en el mundo; es decir: va acompañado de un incremento interior del saber-hacer (aprendizaje) para el agente. Este resultado interno no es otra cosa que la retroalimentación, o feed-back, que toda actividad implica para la esencia de una persona en cuanto que libre. Tanto el procedimiento «ensayo-error-corrección-nuevo ensayo...» como la reiteración de la actividad productiva generan en el productor hábitos prácticos (técnicos y éticos) ${ }^{26}$ que benefician en directo ${ }^{27}$, para el futuro, a su razón práctica en cuanto que productiva.

Por su parte, el término externo reviste la forma de un proceso físico concreto (producto, servicio, o similar) y ordenado según la idea, lo que le otorga una efectividad práctica superior a la que sería la natural de sus componentes físicos sin ordenar. Y, ciertamente, aunque éste no sea el más alto de los resultados de la producción, ha de decirse que es el primero en sentido temporal

25 Lo denominamos «primero» no en sentido jerárquico, sino temporal, porque -como explicamos más abajo- este resultado no es un término que cumpla la intención completa de un proyecto productivo, sino que es seguido por otro que sí la cumple.

26 Los hábitos técnicos tienen que ver con la habilidad para dominar los procesos físicos. Los éticos tienen que ver con la destinación personal, es decir, con la ordenación de la vida al fin último, cfr. Polo, L. (2019b), p. 87: "La ética es necesaria para enderezar el tiempo humano hacia el fin; lo que no mira al destino del hombre no es integralmente moral (con todo, su omisión o conculcación no es constitutivo necesariamente de culpa o de falta). La tesis es: la ética es la organización estricta del tiempo humano respecto de una culminación".

27 Quizás se nos objete que hay «consecuciones» de productos que llevan consigo un grave decrecimiento ético, por ejemplo: si lo producido es droga o armamento. Sin embargo, introducir ideas en el tiempo no es, de suyo, más que obedecer al imperativo categórico moral: «haz» (cfr. Falgueras Salinas, I. y Falgueras Sorauren, I. (2016), p. 46); dependerá de la intención de uso a que se quiera destinar lo introducido el que tal obra sea moralmente buena o mala. Por su parte, lo introducido, o producto externo, en cuanto que físicamente independiente, no es de suyo ni bueno ni malo, sólo aprovechable o no. Pues el que sea bueno o malo no depende de él, sino del uso que con posterioridad se le dé, sobre todo si lo consideramos -como hacemos aquí- en su fase personal-individual. Con todo, del uso hablaremos en el apartado siguiente y en trabajos posteriores. 
bumano, pues su «consecución» condiciona el crecimiento técnico y ético interno de la persona ${ }^{28}$.

De acuerdo con lo anterior, el producto, propiamente hablando, no es más que el resultado externo de la producción ${ }^{29}$. Pero, por ser un primer y necesario punto de apoyo en el que se detiene de modo transitorio la actividad productiva, requiere que fijemos la mirada en él, si queremos entenderla desde su base.

$\mathrm{Al}$ considerar detenidamente el producto, o término externo, hemos de distinguir en él, a su vez, dos componentes. En efecto, aunque durante la introducción del proyecto, incluidos los ensayos prácticos, obtener el producto haya sido el objetivo inmediato, no el último, del proceso productivo, el producto -una vez conseguido, y en cuanto que está dotado de efectividad física propia- goza de cierta independencia respecto de la actividad introductiva que lo ha producido. Y la razón de esa independencia estriba en que el término externo de la producción inicial, por ser físico, tiene las propiedades que le otorga la esencia del mundo, y obedece a las leyes y condiciones naturales que lo rigen, y que son las que le dan efectividad. Éste es un primer componente del producto, que concretamos algo más a continuación.

Por su propia índole, los procesos físicos ${ }^{30}$ son inestables, pues no están quietos ni se detienen, sino que son precedidos y seguidos por muchos momentos procesuales, e incluso por muchos otros procesos. Naturalmente, aun

28 También los ensayos-conato de la actividad introductiva tienen un efecto interno, a saber, el del aprendizaje, sin el cual no se podría corregir lo errado y obtener el producto deseado. Mas, en todo caso, la «consecución» acumula el aprendizaje anterior y le añade el propio, dando lugar a un beneficio interno que es -como decimos- por completo distinto del mero producto o término externo, y forma los hábitos prácticos aludidos. De tal manera que sin la consecución de un producto externo no existen, propiamente, obras humanas ni se adquieren hábitos. Por ejemplo, el hábito de hablar (físicamente) bien sólo se obtiene si se habla bien: los esfuerzos del tartamudo sólo le servirán si consigue hablar bien, y sólo hablando bien de modo repetido adquirirá ese hábito, el cual le permitirá hacerlo sin tales esfuerzos. En este sentido, la consecución de lo externo (producto) «condiciona» el crecimiento interno (hábitos).

29 Decimos «propiamente hablando», porque lo primero que hemos de conseguir los humanos es dominar nuestro cuerpo, aprendiendo a mover nuestras facultades locomotrices (andar, hablar, manejar...), cfr. Falgueras Salinas, I. y Falgueras Sorauren, I. (2016), pp. 47-48. Sin embargo, ese dominio no es una producción, sino una coordinación interna, condición necesaria para poder producir

30 La noción de proceso la utilizamos aquí en sentido amplio. La entendemos como una secuencia de momentos según el antes y el después, prescindiendo de la procedencia, así como de la finalidad del mismo. No es que neguemos ni lo uno ni lo otro, sino que aquí sólo atendemos a su suceder, transcurrir o ir hacia delante. (Diccionario de la Real Academia de la Lengua Española, 23a edición, 2014: https://dle.rae.es: 1. m. Acción de ir hacia delante. 2. m. Transcurso del tiempo. 3. m. Conjunto de las fases sucesivas de un fenómeno natural o de una operación artificial). 
siendo todos inestables, existen unos procesos físicos más rápidos y otros más lentos, según sea su complejidad.

Por el lado, pues, de los componentes físicos (materia ${ }^{31}$ ) de lo «conseguido», caben productos muy inestables y de corta duración en el tiempo; otros menos inestables y de duración media; así como otros semi-estables y de duración larga, tomando siempre como medida el tiempo humano ${ }^{32}$. La producción humana aprovecha los procesos más inestables, como los sonidos, por ejemplo, para establecer la comunicación oral (el habla); o el fuego, para cocinar e incluso para obtener productos de larga duración (hierro, acero, aleaciones); o los explosivos, para horadar montañas, etc. Otros procesos físicos menos inestables, como el barro cocido, la madera, las fibras vegetales, las pieles de animales, etc., los aprovecha para obtener productos de duración media, como las vasijas, los muebles, la ropa, los libros... Y, por último, otros más duraderos, como las piedras, los metales, o las combinaciones de ambos, etc., son aprovechados para conseguir productos de una (relativamente) larga duración, como las Pirámides de Egipto, los pantanos, las casas, los barcos, los aviones... Según lo anterior, por el lado de los componentes físicos del producto se nos ofrece, de acuerdo con su mayor o menor inestabilidad temporal, una diversificación de procesos temporales efectivos, cuyas propiedades ofrecen una gran cantidad de posibilidades para la acción del hombre ${ }^{33}$.

Pero el producto tiene también, precisamente como resultado de la producción, una forma artificial, que no es sino la ordenación de unos procesos físicos obrada desde un proyecto y una elección del agente libre, o persona.

31 La voz «materia» no tiene aquí sentido metafísico, no alude a la causa material, sino al producto, y se opone a la «forma» entendida como configuración externa (artificial) del producto. Tampoco la usamos en sentido meramente metafórico, para significar sólo el asunto o contenido de lo que se trata. Por «materia del producto» entendemos los componentes físico-temporales del mismo; y por «forma del producto» la ordenación que impone el hombre a esos componentes físicos.

32 Una palabra, una canción, una danza, etc., son en su dimensión física procesos rápidos, integrados por movimientos muy inestables, que desaparecen físicamente nada más ser introducidos en el tiempo, y que sólo adquieren cierta estabilidad merced al sentido que el hombre les adjudica. Otros procesos físicos, en cambio, son menos inestables: un plato, un martillo, un coche, etc., tienen también una duración limitada en el tiempo, pero respecto de nuestro tiempo son suficientemente estables. Ejemplos de larga duración podrían ser ciertos monumentos, como las Pirámides egipcias, los acueductos romanos, el Escorial, etc. No hace falta decir que la duración de un meteorito, de un planeta o de una estrella, no siendo ilimitada, es aún mucho más larga, pero no son, de suyo, consecuciones del producir humano.

33 No porque sean inestables los procesos físicos dejan de ser universales. Pensar eso fue uno de los errores básicos de Platón. Los procesos físicos son universales, no particulares, pero con una universalidad física, no meramente pensada, y bien entendido que el universal físico es unum in multis, siendo los «multi» los momentos temporales. Cfr. Polo, L. (2019a), p. 35. 
Ahora bien, al estamparle a un momento -más o menos largo- de un proceso físico la forma u ordenación relativa a la idea y al fin del agente humano, dicho proceso físico adquiere una cierta estabilidad extrínseca a él y relativa a la actividad (teórica y práctica) del hombre. Tal estabilidad, que no detiene la procesualidad natural, es aprovechada por el artificio para ordenar el mundo ${ }^{34}$. Éste es el segundo componente del producto, el componente formal.

$\mathrm{Al}$ terminar de conjuntar de manera satisfactoria los dos componentes del proceso productivo, se lleva a cabo por primera vez el propósito inmediato del proyecto introductivo inherente a la producción. Con la consecución de dicho propósito se ha ganado la efectividad física frente a la mera pensabilidad, que era la situación correspondiente a la pura posibilidad de la idea contenida en el proyecto antes de su introducción en el tiempo físico ${ }^{35}$. Pero, a la vez, con ella se ha introducido en dicho tiempo una ordenación según la idea y el propósito proyectados por el agente.

La conjunción de tales ingredientes aporta la novedad de ofrecer una posibilidad efectiva, un adelanto controlado del futuro, es decir, un proceso físico ordenado según una idea que lo unifica y encauza en la temporalidad extramental, ordenación que antes no existía en el universo ${ }^{36}$. Es a lo que Aristóteles aludía cuando definía lo artificial como aquella entidad física que no tiene el principio de su actuación en sí mismo, sino en el hombre que lo produce, frente a lo natural, que tiene en sí el principio de sus operaciones ${ }^{37}$. La forma

34 "La apertura del tiempo humano hacia el futuro se designa bien con la palabra oportunidades (también podría decirse que el hombre actualiza potencialidades o posibilidades de distinta manera que el movimiento físico)" (Polo, L., 2016a, p. 64).

35 Por eso consideramos como productos los resultados de cualquier acción humana (v.gr.: dar un golpe en la mesa para llamar la atención), puesto que cualquier acción humana, en sentido propio, se hace desde una idea conocida y un propósito voluntario, y de tal modo que, al terminar la producción, aunque el producto resultante (v.gr.: el sonido del golpe dado) no tuviera una clara forma artificial, permita obtener o conseguir dicho propósito. En este sentido, lo que lo convierte en producto no es, pues, tanto la transformación de la materia, (cfr. nota 12), cuanto la integración en la acción humana.

36 O bien no existían en absoluto (como el rayo láser, la televisión, la audición a distancia espacial o temporal, etc., etc.), o bien no existían como posibilidad para el hombre (sintetizar agua, viajar a la luna, etc.).

37 Aristóteles (1831), VI, 4, 1140 a 6-19. Debe notarse que la denominación de «artificial» es acertada para designar la producción y los productos humanos en cuanto que se distinguen de la naturaleza física, pero no de la naturaleza humana, pues como dice Polo: "Lo artificial, que es lo que el hombre hace, es natural para el hombre, pero para el animal no lo es. Hay culturización del sistema nervioso. Hay conexiones neuronales que, si no fuera por el aprendizaje, no se darían. La función constituye el órgano. Es el ejercicio del hombre el que culturaliza el cerebro humano. La fisiología es la base o potencia, pero el cerebro se lo hace el hombre en el ejercicio de su vida” (Polo, L., 2018, p. 45). 
del producto, como ordenación racional de efectividades físicas, lo dota de una finalidad y un sentido humanos.

Para terminar este apartado, se ha de notar que, como el propósito inicial sólo pretende introducir en el tiempo una idea -aunque sea compleja-, en él simplemente se apunta a aprovechar una determinada propiedad, o un grupo de propiedades, de los procesos físicos que nos posibilitará hacer algo también determinado. Mas los procesos ordenados en el producto conseguido tienen en su componente físico más propiedades que las buscadas por el proyecto inicial. Por eso, aunque los productos sean término inmediato de la producción, no son un término final de la actividad productiva, sino que cabe que en ellos mismos nuestro entendimiento descubra otras posibilidades, aunque para ello hará falta una nueva iniciativa del agente, que describiremos en próximos artículos, y a los que da entrada el uso primero de los mismos.

\section{EL TÉRMINO ÚLTIMO DE LA ACTIVIDAD PRODUCTIVA INICIAL: EL USO PRIMERO DEL PRODUCTO}

La primera de las posibilidades efectivas contenidas en el producto es la de su uso, pero para ser precisos es necesario, antes, establecer bien esta última noción.

\section{La noción de uso}

Empecemos por aclarar la noción de uso. Uso es la acción de usar o servirse de $\operatorname{algo}^{38}$. La noción de uso implica, pues, jerarquía: el que usa es superior a lo usado, dispone de lo usado. En el caso del hombre, que es el que aquí consideramos, ese disponer es libre.

En sentido amplio, cabe entender -como hace Tomás de Aquino- que la voluntad usa o dispone de la imaginación cuando la somete a las ideas para formar un proyecto práctico, y cuando la razón práctica ordena a las facultades

\footnotetext{
38 "Hacer servir una cosa para algo" es la primera acepción de la voz «uso» descrita por el Diccionario de la Real Academia Española, antes citado. Ahora bien, el que «hace servir» es el que usa, la cosa es lo usado; por eso resumimos la noción como «servirse de algo», indicando así expresamente la diferencia jerárquica entre ambos términos de la acción. Ya san Agustín resaltó que sólo el hombre, merced a su racionalidad, hace uso de las cosas (cfr. Agustín, S., 1854, cols. 19-20).
} 
locomotrices ejecutarlo ${ }^{39}$. De modo semejante, podrían considerarse como «usos» los distintos ensayos prácticos realizados -por imperio de la razón práctica a las facultades locomotrices, dirigidas por la imaginación y guiadas por la idea contenida en el proyecto- hasta conseguir un producto satisfactorio $^{40}$. Sin embargo, en la medida en que se trata de operaciones que anteceden a la consecución del producto, no son usos del producto, si se entiende por producto, como hemos indicado, sólo el producto conseguido.

El uso del producto es el sentido primigenio de la actividad práctica de «servirse» de algo. Es conveniente recordar que estamos considerando la actividad productiva en su fase personal-individual, que es la que lleva la iniciativa en las dualizaciones manifestativas. Por ejemplo, de lo primero de que nos servimos todos los humanos es del lenguaje en cuanto que es producido por cada uno. Naturalmente, existen también otros sentidos del término «uso», v.gr.: el que lo iguala a las costumbres sociales ${ }^{41}$, el que lo vincula al consumo ${ }^{42}$, y otros más especializados ${ }^{43}$. Pero todos ellos son derivaciones del sentido original, que es, sin duda, el uso de un producto que ba sido producido por uno mismo, ya que en él encuentra primeramente cada hombre su superioridad sobre lo usado, y el inicio de su dominio efectivo del mundo, hasta el punto de que la produc-

39 “Tomás de Aquino afrontó el problema. A la relación entre el querer y el hacer la llama uso, y estima que es un acto de la voluntad que dispone de otras facultades" (Polo, L., 2016b, p. 376). En nuestra propuesta eso equivaldría a considerar como «uso» el dominio del hombre sobre el propio cuerpo, dominio que nos permite andar, hablar, manejar, etc. Sin embargo, como hemos dicho, esos usos serían anteriores al producto, condiciones del producir (cfr. nota 29).

40 Es cierto que los ensayos forman parte de la producción, pero ya antes de estar «conseguida», por lo que no los consideramos usos en sentido estricto.

41 Ortega y Gasset, J. (1964), p. 79.

$42 \mathrm{El}$ uso suele ser asociado al consumo en las sociedades industrializadas: "La palabra consumidor... está pensada desde el ángulo del empresario. Al producir el utensilio, lo destina al ámbito de una acción humana distinta y ulterior, que es indispensable para el mantenimiento de la producción... Pero, en rigor, lo que desde el punto de vista de la actividad industrial se llama consumo, desde la persona que se hace cargo del utensilio debe llamarse justamente uso" (Polo, L., 2019b, p. 66).

43 En referencia al lenguaje, Wittgenstein -por ejemplo- entiende por «uso» el sistema o conjunto organizado (juego lingüístico) de los signos (frases), que es lo que les da vida (cfr. Wittgenstein, L. (1976), pp. 31 ss.). Por otra parte, en la lógica post-fregiana se suele distinguir entre «uso» $\mathrm{y}$ «mención» para separar niveles lingüísticos - por ejemplo, lenguaje objeto y metalenguaje-, y evitar así paradojas -lógicas o semánticas-, cfr. Ferrater Mora, J. (1984), voces «Frege», «Mención» y «Uso». 
ción propia puede ser considerada como el título más obvio de propiedad sobre el producto, y su uso como el ejercicio más natural de la misma ${ }^{44}$.

Es de notar la importancia práctica del uso del producto. La «consecución» del proyecto inicial se refiere a un propósito o fin inmediato del productor, que no es otra cosa sino introducir una idea en el tiempo de modo que ciertas propiedades de los procesos temporales queden organizadas efectivamente según esa idea (un martillo, una conversación, una fuente de energía, etc.). Sin embargo, esta finalidad inmediata, contenida -sí- en la elección del proyecto, no cumple por completo la finalidad última del proceso productivo iniciado, el cual ha terminado directamente en el producto conseguido ${ }^{45}$. Lo explicamos.

Es obvio que, cuando se ha querido obtener un producto, se lo ha querido para hacer algo con él ${ }^{46}$. Ese «hacer algo» puede ser teórico o práctico, pero en el producto está siempre precedido por un «para» que lo subordina, porque el producto no ha sido procurado por razón de sí mismo, sino con otra

44 Para Smith el producto del trabajo es su recompensa natural (cfr. Smith, A., 1904[1994], I, c. 6, 54; c. 8,73 ). Marx critica a los economistas ingleses por reducir el trabajo a una mercancía, objetivándolo (cfr. Marx, K., 1976, vol. 3, p. 163: "la mercancía como valor de cambio es sólo producto, trabajo objetivado"), cuando debiera ser la producción por el hombre de su vida material (cfr. Marx, K., 1972, p. 19). Pero lo cierto es que la producción, o el trabajo, antes de ser la esencia del género humano -como él pretende-, lo es de la de cada persona, y no como su núcleo ontológico o existencial, sino como el ejercicio de una tarea suya, la de la habitación del mundo, constituyendo así el modo más elemental y obvio de apropiación del mundo.

45 "Aquello con lo que ante todo tiene que habérselas el trato cotidiano no son tampoco los utensilios, sino que lo que primariamente nos ocupa (das Besorgte) y está por ende a la mano (Zubandene) es la obra (Werk) misma, lo que en cada caso tiene que ser producido (Herzustellende). Es la obra la portadora de la totalidad remisional dentro de la cual el útil comparece" (Heidegger, M. 1997, p. 97 [SZ, 69-70]). Es importante que el lector advierta la distinción entre los fines humanos y la causa final. La causa final es la que ordena el despliegue de las demás causas mundanas y lo hace de forma estadística, de manera que ella no se «alcanza», sino que está vigente en todos los procesos físicos. En cambio, los fines humanos -a los que dedicaremos atención en próximos trabajos- son: o bien propósitos libres (inmediatos o intermedios) de nuestra voluntad que pretendemos conseguir con la actividad productiva, o bien aquellos fines (últimos o cuasi-últimos) hacia los que nuestra vida ha de orientarse con su libertad.

46 Por ejemplo, en la ciencia empírica, el uso de un producto (v.gr.: un aparato) se hace para comprobar experimentalmente alguna hipótesis. En el arte, el uso (artístico) de un producto se hace al disfrutar de su belleza o al menos de su creatividad expresiva. A los productos artísticos se asemejan aquellos que por razón religiosa, simbólica o afectiva -recuerdo de parientes y amigos, o de acontecimientosquedan separados de un uso productivo. Incluso la filosofía ha de hacer uso de productos lingüísticos para poder encaminar su búsqueda de la verdad, pero al hacerlo no sólo los separa de la producción, sino que prescinde de su sentido práctico y les da uno más profundo. 
finalidad: para servirse o disponer de él ${ }^{47}$. Y eso no es posible hacerlo realmente sin reincorporarlo a la propia actividad productiva humana, porque el producto en sí es inerte o carente de iniciativa, sólo la persona puede disponer de él, y hacerlo útil como producto ${ }^{48}$. Justamente a dicho disponer del producto es a lo que llamamos aquí «uso del producto».

Por tanto, la finalidad última del proyecto productivo es consumada, o llevada por entero a término, cuando el producto conseguido es usado ${ }^{49}$. En este sentido, cabe describir el uso del producto como su aprovechamiento efectivo para el obrar bumano ${ }^{50}$. Es cierto que, lo mismo que pasa con los objetos ${ }^{51}$, los productos atraen tanto nuestra atención que suelen ocultarnos que su verdadera finalidad es el uso efectivo, y nada más que el uso. Y, por eso, con frecuencia nos fascinan tanto que, cuando no nos inducen a creernos omnipotentes ${ }^{52}$, nos

47 "La obra que se quiere producir, y que es el para-qué del martillo, cepillo y aguja, tiene por su parte el modo de ser del útil” (Heidegger, M. (1997), p. 97 [SZ 60-70]). Y ese «para» que precede al producto es la razón de su índole de medio.

48 Podría objetarse que la inteligencia artificial es una clase de productos humanos que puede alcanzar autonomía e incluso superar a la inteligencia humana. Sin embargo, la inteligencia artificial no puede comprender verdades ni asignar fines, por ser un producto pre-programado, carente de vida y, sobre todo, de libertad. Es cierto que puede hacer algunas tareas humanas a gran velocidad, en ayuda de nuestras facultades locomotrices y de nuestra imaginación, e incluso en tareas de cálculo. Pero téngase en cuenta que los números que se utilizan en la computación son los números humanos o conceptoides, no los números físicos (cfr. Polo, L. 2019a, pp. 492-493), y mucho menos aún los del Creador $(S a b 11,20)$. Es, por tanto, una extrapolación de algunas funciones de nuestra inteligencia, un producto útil, pero sin iniciativa propiamente dicha, y que replica la parte mecanizable de nuestra actividad práctica. Tomarse, pues, en sentido literal lo de «inteligencia» sería lo mismo que llamar en serio sabio al tocho de un libro sapiencial, o filosofía al conjunto de páginas -y quien dice páginas, puede decir también sonidos- que la exponen en forma material.

49 En el proyecto inicial no sólo se contiene una idea, o conjunción mental de propiedades de abstractos, sino también una finalidad asociada a ella. Por ejemplo, duro, afilado y ligero para cortar (cuchillo); duro, ancho y pesado para golpear (martillo), etc. Lo mismo que la producción hace efectiva a la idea, el uso hace efectiva la finalidad completa del proceso productivo en su tramo inicial.

50 Aquí por «uso» se entiende el uso del producto, no el imperio de la voluntad sobre las otras facultades, el cual acontece antes de empezar el proceso productivo propiamente dicho, justo para ponerlo en marcha.

51 Nos referimos al cuádruple ocultamiento -ser y esencia del mundo, ser y esencia del hombre- que su presencia nos induce, y que da ocasión a los cuatro modos de abandonar el límite (cfr. Polo, L. 2015a, pp. 294-295.

52 Es la tentación vigente en nuestros días, y en la que caen los que sacralizan la tecno-ciencia, o los que ponen como meta de la vida humana la auto-creación. 
tienta su acumulación, pudiendo suscitar en nosotros una lamentable adicción ${ }^{53}$.

Repetimos. La «consecución» del producto, que es, al inicio, el fin inmediato de la actividad productiva, no es la última meta de ésta. Lo mismo que hemos distinguido entre los conatos productivos y el producto, así ha de distinguirse entre el fin inmediato de la producción y su fin completo. Sólo cuando la actividad humana reincorpora el producto a su acción al usarlo se aprovechan sus propiedades físicas y puede alcanzarse el beneficio buscado en el proyecto, junto con la adquisición de hábitos prácticos adicionales, que mejoran nuestra comprensión del producto, así como nos dotan de un más ágil dominio sobre él.

Pero, al reincorporar el producto a la actividad productiva mediante el primer uso, se abre la puerta a nuevas iniciativas de uso por parte de la libertad de la persona, a través de la sindéresis y de la razón práctica. Tales iniciativas pueden sobrepasar el objetivo inmediato impreso en la forma inicial del producto. Sin embargo, aquí veremos sólo lo relativo al primer uso, dejando para otras publicaciones el considerar los usos subsiguientes.

\section{El uso primero del producto y la noción de utilidad}

Ya hemos visto que el proceso productivo en su inicio funcionaba ensayando y corrigiendo hasta conseguir un producto que permita llevar a la práctica el proyecto deseado. Tales «conatos de uso» son anteriores a la «consecución» del producto. Pero, como también hemos señalado, el proceso productivo no acaba en el producto, sino que es completado (relativamente) por su uso. Calificamos como «primero» aquel uso de cualquier producto que se corresponde estrictamente con el propósito o fin terminal que guió su producción. No se trata, por tanto, de una denominación en sentido temporal, ni tampoco jerárquico, pues afecta sólo a la finalidad humana que ha quedado incorporada en la forma del producto. «Primero» tiene, pues, aquí un sentido

53 Adorar la obra de las propias manos es el gran pecado, la idolatría (Sab 13, 10). Pero esa «adoración» puede revestir diversas formas. El consumismo es una de las formas que adopta esta nueva esclavitud: "En efecto, este superdesarrollo, consistente en la excesiva disponibilidad de toda clase de bienes materiales .... fácilmente hace a los hombres esclavos de la «posesión»... sin otro horizonte que la multiplicación o la continua sustitución de los objetos que se poseen por otros todavía más perfectos. Es la llamada civilización del «consumo» o consumismo, que comporta tantos «desechos»o «basuras»" (Juan Pablo II, 1987, n. 28). 
antropológico y práctico, pero restringido a lo personal-individual: es el uso directo de un producto que ha sido producido por uno mismo ${ }^{54}$.

Es este uso primero del producto el que lo convierte, por primera vez, en instrumento o utensilio ${ }^{55}$, porque los ensayos anteriores sólo atendían a conseguir la eficacia del producto en sí mismo, mientras que ahora se procura aprovechar dicha eficacia, ya incorporada al producto, de manera que nos sirva para hacer lo que nos propusimos inicialmente. Sin embargo, acerca de las características de los instrumentos, o medios, nos ocuparemos en un trabajo posterior.

Y, a su vez, la noción de uso primero del producto nos va a permitir establecer el sentido básico de la noción de utilidad, pues si la voz «uso» admite muchos sentidos, también la de «utilidad» los tiene. Esta multiplicidad de acepciones se pone de manifiesto, por ejemplo, en la propia ciencia económica, pues dicho término ha sido entendido de distintas formas según ha ido evolucionando históricamente la comprensión de los problemas en los que la utilidad ha tenido alguna relevancia.

Concretamente, según propone Stigler ${ }^{56}$, los aludidos han sido dos grandes grupos de problemas: los relativos a lo que se conoce como economía del bienestar $^{57}$, y los relacionados con la explicación del precio o valor de los bienes. Siguiendo a este autor, y por motivos semejantes a los que él ofrece, vamos a considerar, muy brevemente, sólo el segundo grupo de problemas.

54 Por eso nuestra consideración, aquí, difiere de la de Heidegger. Para éste, nosotros estamos ya instalados en un "mundo pragmático" en el que los entes útiles nos salen al encuentro. Y eso es verdad, pues en buena parte vivimos en y mediante productos humanos que nos anteceden. Pero nosotros, antes que al conjunto de los útiles, estamos atendiendo al modo en que cada hombre -incluidos los primeroshace surgir el mundo pragmático que después lo rodea. Es decir, lo consideramos antes de que nos salga al encuentro, justo en el modo en que va saliendo de nuestras manos.

55 Heidegger llama utensilio $(Z e u g)$ al ente que nos sale al encuentro (begegnende) cuando nos ocupamos con cuidado o angustia (Besorgen) del trato con el mundo (cfr. Heidegger, M. (1997), p. 96 [SZ 68]). Nosotros llamamos utensilio o útil al producto en cuanto que es usado, y, en especial -aquí-, la primera vez, porque en ella es aprovechada la utilidad a que apuntaba el proyecto de producción. Como veremos en otro artículo, los útiles admiten, además, usos derivados, o secundarios, no contemplados en su fabricación original.

56 Cfr. Stigler, G. J. (1950a, 1950b).

57 Para una explicación más detallada de la influencia del utilitarismo como corriente filosófica en la economía en general y en la economía del bienestar en particular, cfr. Welch, C. (2018), y Riley J. (2018), respectivamente. 
Como señala Collinson Black $^{58}$, los primeros economistas, principalmente los clásicos, equipararon la noción de utilidad a la de "deseabilidad" de los bienes, lo que le dió un tono más subjetivo que el que tiene en el inglés común -y que se parece a la que nosotros vamos a proponer más abajo-. Frente al interés de los economistas clásicos en explicar el precio natural de los bienes -determinado por el coste de producción- los economistas posteriores se interesaron más en explicar el precio de mercado de los mismos, lo que hizo que su atención se centrara en entender la función de demanda, y con ella, la noción de utilidad. La revolución marginalista logró fundamentar de modo claro dicha función de demanda en la noción de utilidad, aunque no todos los marginalistas la entendieran como mero placer. Entre ellos fue Jevons quien -al adherirse explícitamente a la propuesta utilitarista de Bentham ${ }^{59}$ - equiparó entre sí estas dos últimas nociones ${ }^{60}$, concibiendo de este modo la utilidad como medible y cuantificable. Posteriormente, como señala Stigler ${ }^{61}$, en su intento de obtener teorías cada vez más generales, los economistas empezaron a poner en duda la anterior equiparación de utilidad y placer. En concreto, las aportaciones de Fisher y Pareto, al sustituir con las curvas de inferencia el papel que la función de utilidad tenía en la fundamentación de la función de demanda, abrieron el camino para que se abandonase la concepción hedonista de la utilidad. Aportaciones posteriores como la de Hicks y Allen ${ }^{62}$, entre otros, ayudaron a establecer la concepción de la utilidad que prevalece hoy en día en la teoría del consumidor, y según la cual ella se reduce a una representación ordinal de la escala jerárquica de preferencias del consumidor ${ }^{63}$.

Fuera de la ciencia económica, hay otras nociones más extremosas de la utilidad, como es la que ofrece, por ejemplo, Nietzsche, quien la considera

58 Cfr. Collinson Black R. D. (2018). Hay que señalar que existen economistas clásicos, como A. Smith, en los que, aunque se entiende de modo prevalente la utilidad en sentido subjetivo (deseabilidad), en algunas ocasiones, no obstante, la entienden como "valor real" (cfr. Bowley, M. (1973), pp. 137-138), es decir, como la capacidad de producir un efecto beneficioso para quien los usa.

59 Cfr. Jevons, W. S. (1957), p. 28.

60 Jevons, siguiendo a Senior (cfr. Senior, N. W. (1821[1965]), p. 6), define la utilidad como la cualidad de proporcionar placer o evitar dolor (Cfr. Jevons, W. S. 1965, p. 2). Incluso Hume y Smith -que lo cita- adelantaron en cierto sentido esta noción de utilidad (cfr. Smith, A. 1976[1982], p. 179). También Stuart Mill había propuesto algo muy parecido (cfr. Mill, J. S. 1969, p. 209).

61 Cfr. Stigler, G. J. (1950b), pp. 377-383.

62 Cfr. Hicks, J. R. y Allen, R. G. D. (1934).

63 Cfr. Dyke, C. E. (1981), pp. 48 ss. Lo que acontece, según Kapteyn A. (1985), p. 2, es que se deja de tomar como base del análisis la noción de utilidad. En la moderna teoría económica, según él, la utilidad deja de ser el concepto primitivo y pasa a ser la ordenación de preferencias, de modo que la utilidad deja de ser interpretada cuantitativamente, y adquiere un significado cualitativo. 
como un valor de esclavos ${ }^{64}$. En el cabo opuesto, el pragmatismo americano eleva, en términos generales, la utilidad al rango de criterio de verdad ${ }^{65}$. Y, de modo más equilibrado, en la teoría de los valores, la utilidad es considerada como uno de ellos, aunque ciertamente como el menor de todos ${ }^{66}$.

Es bastante obvio que todas estas maneras de entender la utilidad son posteriores a la formación de su noción, pues implican una asociación, o al menos una comparación, de nociones. Por nuestra parte, entendemos por $u t i-$ lidad primera el provecho que se obtiene del uso directo de un producto pro$\mathrm{pio}^{67}$. Naturalmente, ese aprovechamiento se corresponde con el propósito que el productor tenía, y, en ese sentido, lo satisface. Pero la satisfacción de que se habla no tiene que ver necesariamente con el placer subjetivo, ni menos aún con la felicidad, sino con la eficacia real del producto respecto del fin que al producirlo se buscaba ${ }^{68}$. Por ejemplo, la utilidad de un cuchillo es que sirva

64 Cfr. Nietzsche F. (2000), $\$ 720$, p. 483; $\$ 752$, p. 499; \$861, p. 578. Ese juicio despectivo (cfr. Nietzsche F. (1972), \$225, p. 171-172) es relativo a la utilidad económica y pragmatista, pero -eso no obstante- la interpretación nietzscheana de la verdad como mero instrumento de la voluntad de poder ha inspirado a Rorty, representante contemporáneo del pragmatismo americano (cfr. Rorty, R. 1989, p. 27).

65 Aunque Lovejoy A. O. (1908a, 1908b) recogió hasta trece tendencias en el pragmatismo americano, se puede decir que una de las notas comunes a dicha corriente de pensamiento es el reducir-de una u otra forma- la verdad a utilidad. Eso es así desde James -para quien es lo mismo decir que una idea es útil porque es verdadera, o que es verdadera, porque es útil (cfr. James, W. (1907), pp. 219-220)hasta Rorty R. (1989), quien desecha considerar la verdad y lo verdadero en sentido filosófico (p. 8), por ser la filosofía la mera construcción de un lenguaje que no es útil para la tarea de la autocreación práctica del hombre por el hombre (p. XV), al responder sólo a intereses particulares, y no también públicos (p. 118), cuya utilidad no puede ser comprobada más que retrospectivamente (p. 55). El pensamiento de estos autores no es consistente, pues antes de ser útil (o no) es preciso que el lenguaje sea entendido como verdadero; más aún, el simple acto de afirmar algo al hablar implica que se propone como verdadero. Por ejemplo, afirmar que el lenguaje filosófico no es útil, como hace Rorty, contiene la pretensión de que se entienda como una tesis verdadera.

66 En la escala apriorística de los valores materiales de Scheler, compuesta por cuatro grados, el primero y más bajo corresponde a la utilidad en el mismo sentido que le da el utilitarismo (placer y dolor sensible), por debajo de los valores vitales, de los valores espirituales y de los valores puramente personales o sagrados (cfr. Scheler, M. 2001, pp. 173-179).

67 De modo parecido lo entendía Malthus, T. R. [1827(1986)], p. 234, cuando definía la utilidad (de un objeto) como la cualidad (que tiene) de prestar algún servicio o beneficio a la humanidad. También Say la entendió así (cfr. Say, J.-B. 1821[1971], p. 62).

68 Según explica Clark, J. M. [1936(1967)], pp. 25-26, los economistas clásicos sobrevaloraban la utilidad, porque, al entender el trabajo únicamente como esfuerzo penoso -que contradecía su búsqueda del placer o bienestar-, pasaban a considerar el producto y su utilidad como la única recompensa por dicha penalidad. Pero si se concibe el trabajo cual tarea propia del hombre, y no como esfuerzo necesariamente costoso, la utilidad (felicitaria) del producto pasa a ocupar un puesto secundario. Algo semejante, aunque en otro contexto, sugiere Polo cuando dice que el hombre no está hecho para autopremiarse con el placer, sino para acrecer su capacidad vital con el trabajo (cfr. Polo, L. 2015c, pp. 352-353). 
para cortar. El placer se puede asociar con la carne del animal que se ha conseguido comer después de despellejarlo. Pero el cuchillo no produce placer, sólo es útil, y éste es el sentido elemental y primero de utilidad: la eficacia real del producto en relación con el propósito inicial del proyecto productivo ${ }^{69}$.

Este sentido obvio y primero de la utilidad no excluye otros sentidos posibles, aunque derivados, como algunos de los mencionados más arriba, pues habiendo sido formado a partir del primer uso del producto, y existir usos más complejos, el sentido señalado no impide otros sentidos de la utilidad, tan sólo señala algo que es común a todos, y que permite separarla de exclusiones arbitrarias. Pues la utilidad, al igual que la propia actividad práctica, no es algo ni puramente subjetivo ni puramente objetivo, como tampoco es algo exclusivamente individual ni exclusivamente social, sino que reúne todos esos aspectos.

\section{CONCLUSIÓN}

El objetivo de este trabajo era investigar -como continuación del estudio por separado de sus dos polos (escasez y riqueza)- el despliegue conjunto de la dualización productiva normal, pero sólo en su primer tramo. Y hemos visto que dicho despliegue comienza con la ejecución del proyecto elegido mediante la operación de las facultades locomotrices, dirigidas por la imaginación bajo la guía de las ideas, y puestas en marcha por el imperium de la razón práctica.

$\mathrm{Al}$ prestar atención al primer tramo del proceso productivo, la noción de producción se ha hecho más precisa, aunque sin contradecir su sentido amplio. Ella muestra que es menos extensa que la dualización esencial y que la actividad práctica humana, las cuales, no obstante, reciben el calificativo de «productivas» justamente porque, en su sentido más estricto -aunque todavía no en el económico-, la introducción de una idea en el tiempo, o la ordenación de los movimientos físico-temporales según una idea, es la base de todas ellas, siendo el modo en que todo obrar humano tiene su comienzo.

En este primer tramo de la dualización productiva se han de coaptar lo atemporal (ideas y voliciones) de nuestra alma con la temporalidad física del mundo. Los procesos mediante los cuales se coordinan ambos extremos, atem-

69 Cfr. la referencia al sentido primero y normal, en inglés, de la palabra «utilidad» que hace Collinson Black, R. D. (2018), p. 14219. 
poral y temporal, en apariencia incompatibles, se caracterizan por revestir la forma del método «ensayo-error-corrección del ensayo...», porque "para saber cómo se bace lo que se quiere hacer es preciso hacer lo que se quiere saber". Hablamos de los procesos productivos básicos. En ellos intervienen, al menos, cuatro potencias humanas: (i) dos espirituales, (i.a) la inteligencia y (i.b) la voluntad, y (ii) dos corporales, (ii.a) la sensibilidad -externa (cinco sentidos) e interna (imaginación, memoria y cogitativa) - y (ii.b) las facultades locomotrices (manos, brazos, piernas, lengua...). Por eso, la dualización en que ellas se integran se denomina productiva normal, al ser la, humanamente, más equilibrada y aquella por la que comienza toda actividad humana en el mundo.

Ahora bien, la conjunción de todas esas potencias en el proceso productivo lleva tiempo. El inevitable gasto de tiempo tiene su origen en la inclusión de procesos físico-temporales en el proceso productivo humano. Pero la posibilidad de tal inclusión es abierta por la diferencia jerárquica que existe entre lo atemporal y lo temporal en el hombre. De modo que, si la imaginación sirve de medianera por parte del cuerpo, es la superioridad de la inteligencia y de la voluntad la que admite, por parte del alma, mezclarse con el retraso temporal sin sucumbir a él, sino sacándole provecho.

$\mathrm{Y}$ justo porque se le puede sacar provecho, cuando la introducción de la idea en el tiempo -tras los necesarios ensayos y correcciones- obtiene un término satisfactorio de acuerdo con el propósito inicial del proceso, entonces tenemos la «consecución» del proceso productivo, que es su primer resultado satisfactorio.

Sin embargo, la «consecución» no es simple, antes bien, tiene dos vertientes: una externa y otra interna. La externa es lo que se suele llamar estrictamente el producto; la interna son los hábitos técnicos y morales que al ejecutar la idea se van adquiriendo. Tales hábitos no son otra cosa que el efecto retroactivo que toda actividad de una persona libre tiene sobre ella misma, y que en la esencia del hombre abre la vía por la que puede crecer ilimitadamente.

Por su parte, el resultado externo de la «consecución», el producto, tiene, a su vez, otros dos componentes: uno material y otro formal. El componente material está constituido por los procesos puramente físicos que se contienen en él; el formal está constituido por los abstractos ideales -mediados imaginativamente- que han sido plasmados en la materialidad del producto en forma de ordenación artificial. 
Por ser el producto el primer resultado en sentido temporal bumano -pues su «con-secución» condiciona el posible crecimiento técnico y ético (interno) de la persona-, así como por entrar más directamente en la línea temática (la actividad económica) de nuestra investigación, nos hemos detenido algo más en la descripción de estos dos componentes del producto.

El componente material es inestable, dado que todo lo que existe en el mundo físico está en movimiento. Pero, eso no obstante, existen unos movimientos o procesos físicos más rápidos que otros: unos son muy inestables, otros menos inestables, y otros semi-estables; y tales diferencias puede ser aprovechadas por el hombre, porque el tiempo humano está articulado desde la atemporalidad. Dicho aprovechamiento consiste en obtener eficacia física frente a la pura pensabilidad de nuestros meros proyectos.

El componente formal, que resulta de la inserción en los procesos físicos de una ordenación extrínseca impuesta por la idea y la volición humanas, otorga al producto cierta independencia de la causa final mundana, así como una estabilidad relativa a nuestros proyectos e intenciones, la cual nos permite dominar las inestabilidades físicas.

La conjunción de ambos factores en el producto tiene como aportación el ofrecer posibilidades efectivas para el obrar bumano, en las que se anticipan procesos físicos futuros, que todavía no existen y que están dirigidos por una idea y una intención que los aúna y conduce en la temporalidad extramental. La primera de esas posibilidades humanas efectivas es el uso del producto, pues con él se cumple la intención inicial que llevó a su producción. Por ello, le hemos prestado especial atención.

Hemos empezado por sentar la noción de uso como la acción de servirse o disponer de algo. El implícito de dicha noción es la existencia de una diferencia jerárquica entre quien ejerce la acción y aquello de que se sirve. Y, si bien dicha noción admite varios sentidos diferentes, el primero de ellos -antropológicamente hablando- es el que se refiere en directo al producto, dado que lo primero que usa -en sentido estricto- cada hombre, como hombre, son sus propios productos.

De ahí que hayamos centrado nuestra atención, por el momento, en el uso primero del producto. Y de él hemos averiguado que es la consumación de la finalidad completa contenida en el proyecto de producción, puesto que cuando se quiere producir algo se lo quiere para bacer algo con lo producido, y eso es lo que se lleva a cabo al usarlo. En tal sentido, el uso primero del producto puede ser descrito como su primer aprovechamiento efectivo para el obrar bumano. 
Sin embargo, debe entenderse que todo uso, incluido el primero, reintroduce el producto en la actividad humana, pues - una vez producido- el producto tiene una entidad propia e independiente, aunque inerte. Debido a dicha entidad propia acontece comúnmente que los productos, fruto del ingenio humano, reclaman tanto nuestra atención que llegan a fascinarnos, haciéndonos olvidar que su verdadero objetivo es el uso, y nos tientan sea con la sugerencia quimérica de autoproducirnos, sea con el deseo de acumularlos.

Finalmente, del primer uso del producto hemos inducido el sentido básico de la noción de utilidad, el cual se reduce a la eficacia real que se obtiene del uso primero del producto. Precisamente porque está extraído del primer uso del producto, pero existen usos posteriores, este sentido no excluye otros sentidos más complejos de la utilidad, tan sólo señala algo que es común a todos, y que permite separar su comprensión de extremos arbitrarios. A estudiar los usos ulteriores del producto, y el mundo que de ellos deriva, estarán dedicados los próximos pasos de nuestra investigación, que serán objeto de artículos posteriores. 


\section{BIBLIOGRAFÍA}

Agustín, San (1845), De diversis quaestionibus LXXXIII, q. XXX, Patrologiae Cursus Completus, Series I (Ecclesiae Latinae), J.-P. Migne accurante, Parisiis, vol. XL, cols. 11-100.

Aristóteles (1831), Ethica Nicomachea, en Aristotelis Opera, I. Bekker, Akademia Regia Borussica, Berolini, vol. Alterum, pp. 1094-1181.

Bowley, Marian (1973), Studies in the History of Economic Theory before 1870, Palgrave Macmillan, London.

Clark, John Maurice [1936(1967)], Preface to Social Economics, Essays on Economic Theories and Social Problems, Augustus M. Kelley, Nueva York.

Collison Black, Robert Denis (2018), "Utility”, en The New Palgrave Dictionary of Economics, Palgrave Macmillan, Londres (3 ${ }^{\text {a }}$ ed.), pp. 14219-14224.

Dyke, Charles E. (1981), Philosophy of Economics, Prentice-Hall, Inc., Englewood Cliffs.

Espinosa, Benito (1972), Ethica more geometrico demonstrata, Spinoza Opera im Auftrag der Heidelberger Akademie der Wissenschaften, herausgegeben von Carl Gebhardt, Carl Winters Universitätsverlag, Heidelberg. Auflage. Band II, pp. 41-308.

Falgueras Salinas, I. (2020), “Todo éxito es prematuro”, Studia Poliana, 22, pp. 35-60.

Falgueras Salinas, I. (2021), "El movimiento circular y su función en el cosmos”, en F. Rodríguez Valls y J.J. Padial (eds.), Ciencia y Filosofía, Estudios en bomenaje a fuan Arana, Editorial Thémata, Sevilla, 2021, vol. I, pp. 295-310.

Falgueras Salinas, I. y Falgueras Sorauren, I. (2016), "Man as Dualizing Being. The Remote Anthropological Basis of Economic Activity (II)", Fournal of Polian Studies, 3, pp. 29-54.

Falgueras Salinas, I. y Falgueras Sorauren, I. (2020), "La dualización productiva normal: precisiones y ampliación. Bases antropológicas próximas de la actividad económica (I)”, Studia Poliana, 22, pp. 133-193.

Falgueras Salinas, I. y Falgueras Sorauren, I. (2022a), "El despliegue de la dualización productiva normal: consideración de su polo superior (riqueza). Bases antropológicas próximas de la actividad económica (II)", Revista Estudios Filosóficos Polianos, en impresión. 
Falgueras Salinas, I. y Falgueras Sorauren, I. (2022b), "Las dualizaciones en L. Polo. El juego de la imaginación en el inicio del despliegue conjunto de la dualización productiva normal. Bases antropológicas próximas de la actividad económica (III)", Miscelánea Po-liana, Serie Filosofía, 21, pp. 107-123. Disponible en: https://www.leonardopolo.net/wp-content/uploads/2021/ 12/MP71-7.pdf

Ferrater Mora, José (1984), Diccionario de Filosofía, Alianza Editorial, Madrid, 4 vols. ( $5^{\text {a }}$ edición).

Heidegger, Martin (1997), Ser y Tiempo, trad. J. E. Rivera, Editorial Universitaria, Santiago de Chile. [En las citas incluimos la paginación de Sein und Zeit, M. Niemayer Verlag, Tübingen, 2001 (18 ed.)].

Hicks, John R., y Allen, Roy G. D. (1934), "A reconsideration of the Theory of Value. Part I", Economica, 1, n 1, pp. 52-76.

Inciarte, Fernando (1974), El reto del positivismo lógico, Rialp, Madrid.

James, William (1907), Pragmatism, A New Name for Some Old Ways of Thinking, Longmans, Green and Co., New York, etc.

Jevons, William S. (1957), The Theory of Political Economy, Kelley \& Millman Inc. ( $5^{\text {a }}$ ed.).

Jevons, William S. (1965), The principles of Economics and other papers, New York, Augustus M. Kelley.

Juan Pablo II (1987), Sollicitudo rei socialis. Disponible en: https://www.vatican.va/content/john-paul-ii/it/encyclicals/documents/hf_jpii_enc_30121987_sollicitudo-rei-socialis.html

Kapteyn, Arie (1985), "Utility and economics", De Economist, 133, n 1, pp. 1-20.

Lovejoy, Arthur O. (1908a), "The Thirteen Pragmatisms (I)”, fournal of Philosophy, Psychology and Scientific Methods, 5, n ${ }^{\circ}$ 1, pp. 2-12.

Lovejoy, Arthur O. (1908b), "The Thirteen Pragmatisms (II)", Fournal of Philosophy, Psychology and Scientific Methods, 5, n 2, pp. 29-39.

Malthus, Thomas R. [1827(1986)], Definitions in Political Economy, Augustus M. Kelley, Fairfield.

Marx, Karl (1976), Elementos fundamentales para la crítica de la Economía Política (Grundrisse), trad. P. Scaron, Edit. Siglo XXI, Madrid, 3 vols. 
Marx, Karl y Engels, Friedrich (1972), La Ideología alemana, trad. W. Roces, Ediciones Pueblos Unidos y Ediciones Grijalbo, Montevideo y Barcelona ( $4^{a}$ ed.).

Mill, John Stuart (1969), Utilitarianism, en The collected works of J. S. Mill, Essays on Ethics, Religion and Society, University of Toronto Press, Routledge \& Kegan Paul, Toronto, vol. X.

Nietzsche, Friedrich (1972), Más allá del bien y del mal, trad. A. Sánchez Pascual, Alianza Editorial, Madrid.

Nietzsche, Friedrich (2000), La voluntad de poder, trad. A. Froufe, Ediciones Edaf, Madrid.

Ortega y Gasset, José (1964), El hombre y la gente, Ediciones Revista de Occidente, Madrid ( $4^{\mathrm{a}}$ ed.).

Platón (1901[1979]), Symposium, Platonis Opera, Tomus II, J. Burnet (ed.), Oxford 1901, 172a-223d.

Polo, Leonardo (2015a), El acceso al ser, Obras Completas, Serie A, Eunsa, Pamplona, vol. II.

Polo, Leonardo (2015b), La originalidad de la concepción cristiana de la existencia, Obras Completas, Serie A, Eunsa, Pamplona, vol. XIII, pp. 187-378.

Polo, Leonardo (2015c), Filosofía y Economía, en Obras Completas, Serie A, Eunsa, Pamplona, vol. XXV.

Polo, Leonardo (2016a), Quién es el hombre, Obras Completas, Serie A, Eunsa, Pamplona, vol. X, pp. 21-211.

Polo, Leonardo (2016b), Antropología trascendental, Obras Completas, Serie A, Eunsa, Pamplona, vol. XV.

Polo, Leonardo (2018), Lecciones de Ética, Obras Completas, Serie A, Eunsa, Pamplona, vol. XI, pp. 15-138.

Polo, Leonardo (2019a), Curso de teoría del conocimiento IV, Obras Completas, Serie A, Eunsa, Pamplona, vol. VII.

Polo, Leonardo (2019b), El hombre en la historia, Obras Completas, Serie A, Eunsa, Pamplona, vol. XVIII, pp. 21-136.

Rasmussen, Svend (2013), Production Economics, The Basic Theory of Production Optimisation, Springer, Heidelberg, etc. 
Riley, Jonathan (2018), "Utilitarianism and Economic Theory", en The New Palgrave Dictionary of Economics, Palgrave Macmillan, Londres ( $3^{\mathrm{a}}$ ed.), pp. 14205-14218.

Rorty, R. (1989), Contingency, irony and solidarity, Cambridge U. Press, Cambridge, New York, etc.

Say, Jean-Baptiste [1821(1971)], A treatise on Political Economy or the Production, Distribution and Consumption of Wealth, Augustus M. Kelley, New York.

Scheler, Max (2001), Ética. Nuevo ensayo de fundamentación de un personalismo ético, trad. de Hilario Rodríguez Sanz, Ed. Caparrós, Madrid (3ª ed.).

Senior, Nassau W. [1836(1965)], An Outline of the Science of Political Economy, Augustus M. Kelley, Nueva York.

Smith, Adam [1976(1982)], The theory of the Moral Sentiments, en The Glasgow Edition of the Works and Correspondence of Adam Smith, Liberty Fund, Indianapolis.

Smith, Adam [1904(1994)], An Inquiry into the Nature and Causes of the Wealth of Nations, The Modern Library, Nueva York ( $5^{\mathrm{a}} \mathrm{ed}$. .).

Stigler, George J. (1950a), "The Development of Utility Theory. I", Journal of Political Economy, 58, n 4, pp. 307-327.

Stigler, George J. (1950b), "The Development of Utility Theory II", Fournal of Political Economy, 58, n 5, pp. 377-396.

Tomás de Aquino (1980), Summa Theologiae, S. Thomae Opera, curante R. Busa, Frommann-Holzboog, Stuttgart-Bad Cannstatt, vol. 2.

Von Mises, Ludwig(1996), Human Action. A Treatise on Economics, Fox \& Wilkes, San Francisco.

Welch, Cheryl B. (2018), "Utilitarianism”, en The New Palgrave Dictionary of Economics, Palgrave Macmillan, Londres ( $3^{\text {a }}$ ed.), pp. 14195-14204.

Wicksell, Knut (1938), Lectures on Political Economy, Routledge \& Sons, Londres, 2 vols.

Wittgenstein, L. (1976), Cuadernos azul y marrón, trad. F. Gracia Guillén, Editorial Tecnos, Madrid ( $2^{\mathrm{a}} \mathrm{ed}$.). 